\title{
INTEGRAL MEANS ON RADIALLY WEIGHTED SPACES OF ANALYTIC FUNCTIONS
}

\author{
MATS ERIK ANDERSSON
}

(Received 7 May 1997; revised 11 February 1998)

\author{
Communicated by P. C. Fenton
}

\begin{abstract}
Hilbert spaces of analytic functions generated by rotationally symmetric measures on disks and annuli are studied. A domination relation between function norm and weighted sums of integral means on circles is developed. The function norm and the weighted sum take the same value for a specified class of polynomials. This class can be varied according to two parameters. Parts of the construction carry over to other Banach spaces of analytic or harmonic functions. Counterexamples illuminating properties of the complex method of interpolation appear as a byproduct.
\end{abstract}

1991 Mathematics subject classification (Amer. Math. Soc.): primary 30H05; secondary 30E05, 46B70. Keywords and phrases: Bergman spaces, interpolating polynomials, Gauss-Jacobi quadrature, moment sequence, complex method of interpolation.

\section{Introduction}

Let $\Omega_{1}$ be a generalized circular annulus $A\left(R_{1}, R_{2}\right)=\left\{z \in \mathbb{C} ; R_{1}<|z|<R_{2}\right\}$. We allow $R_{1}<0$ as well as $R_{2}=+\infty$ in order to represent disks centered at the origin or at the point at infinity. Let $\Omega$ be the union of $\Omega_{1}$ with any or none of $\left\{|z|=R_{1}\right\}$, $\left\{|z|=R_{2}\right\}$, except that $R_{2}=+\infty$ may not be used. On $\Omega$ we take a fixed but initially arbitrary rotationally symmetric measure $\mu$. The centre of rotation is the origin. Consider $\Gamma_{\mu}=\left\{k \in \mathbb{Z} ; \int_{\Omega}\left|z^{k}\right|^{2} d \mu(z)<\infty\right\}$ and let $L=\inf \Gamma_{\mu}, K=\sup \Gamma_{\mu}$. In case $L<0$ we impose the condition that $0 \notin \Omega$. At times the interval $I_{\Omega}=\Omega \cap[0, \infty[$ will be used.

This paper is concerned with the generalized Bergman spaces $L_{a}^{2}=L_{a}^{2}(\Omega, \mu)$ consisting of the closure in $L^{2}(\Omega, \mu)$ of $\left\{\phi_{k}: \phi_{k}(z)=z^{k}, k \in \Gamma_{\mu}\right\}$. Due to rotational symmetry the $\phi_{k} \mathrm{~s}$ are orthogonal and $L_{a}^{2}$ consists entirely of analytic functions.

(C) 1998 Australian Mathematical Society 0263-6115/98\$A2.00+0.00 
The norm is $\|f\|=\left(\int_{\Omega}|f(z)|^{2} d \mu(z)\right)^{1 / 2}$ and the natural inner product is used. Observe that the Fock-Bargmann space $L_{a}^{2}\left(\mathbb{C}, e^{-|z|^{2}} d A(z)\right)$, the common Bergman spaces $L_{a}^{2}\left(\mathbb{U},|z|^{\alpha} d A(z)\right)$ as well as spaces containing functions with poles in the hole interior to $\Omega$ are contained in the setting above.

The rotational symmetry of $\mu$ allows a decomposition $d \mu=d \theta \otimes d \nu_{1}=d \theta \otimes d \nu_{2}$ in the sense that

$$
\int_{\Omega}|f(z)|^{2} d \mu(z)=\iint_{0}^{2 \pi}\left|f\left(r e^{i \theta}\right)\right|^{2} \frac{d \theta}{2 \pi} d \nu_{1}(r)=\iint_{0}^{2 \pi}\left|f\left(\sqrt{x} e^{i \theta}\right)\right|^{2} \frac{d \theta}{2 \pi} d \nu_{2}(x),
$$

where $v_{1}$ is supported in $I_{\Omega}$ and $v_{2}$ in $J_{\Omega}=\left\{x \geq 0 ; \sqrt{x} \in I_{\Omega}\right\}$. Notice that there is a bijective correspondence $\mu \leftrightarrow \nu_{2}$ through the change of variables $z=\sqrt{x} e^{i \theta}$. We will make an assumption throughout this paper, that $\nu_{2}$ is not supported on finitely many points only.

This suggests the integral mean mapping $M_{f}: L_{a}^{2} \rightarrow L^{2}\left(I_{\Omega}, \nu_{1}\right)$ given by

$$
M_{f}(r)=\left\{\int_{|z|=r}|f(z)|^{2} \frac{d \theta}{2 \pi}\right\}^{1 / 2} .
$$

Each $f \in L_{a}^{2}$ can be expanded $f(z)=\sum_{n=L}^{K} a_{n} z^{n}$, whence $M_{f}(r)^{2}=\sum\left|a_{n}\right|^{2} r^{2 n}$. It follows that

$$
\|f\|=\left\{\sum_{n=L}^{K}\left|a_{n}\right|^{2} \int_{J_{\Omega}} x^{n} d \nu_{2}(x)\right\}^{1 / 2} .
$$

In [A] the author used the above interpretation for the weighted Bergman spaces with $d \mu_{\alpha \beta}(z)=|z|^{2 \beta}\left(1-|z|^{2}\right)^{\alpha} d A(z)$, where $d A$ is area measure on the unit disk. A central result of $[\mathrm{A}]$ was the following theorem.

THEOREM A. Let $k \geq 0, l \geq 1$ be integers, $\alpha, \beta>-1$. Then there exist positive numbers $A_{j}$ and $r_{j}$ such that for every $f \in L_{a}^{2}\left(U, \mu_{\alpha \beta}\right)$ the following inequality holds.

$$
\sum_{j=1}^{l} A_{j} \int_{|z|=r_{j}}|f(z)|^{2} \frac{d \theta}{2 \pi} \leq\|f\|^{2} .
$$

In addition, equality holds precisely for all $f(z)=a_{k} z^{k}+\cdots+a_{k+2 l-1} z^{k+2 l-1}$. The numbers $r_{j}^{2}$ are the zeros of the lth Jacobi polynomial $P_{l}^{(\alpha . \beta)}(2 x-1)$.

It is the purpose of this paper to expand this result to the general setting. The complete counterpart is stated in section 3. A key technique in [A] was to use Rodrigue's formula for the Jacobi polynomials. That formula is not available in 
general, so a different approach will be taken. As a notable side effect we will find that the integral means form a partial order of norms as the number of circles increases. For example, the mean along one circle does not exceed a particular weighted combination of integral means along two other circles. Finally, the last two sections deal with examples showing the shortcomings of the present technique if we on one hand study harmonic functions and on the other hand we want to look at the general $L^{p}(\mu)$-version of Bergman spaces.

The original motivation for this paper was to explain the observation made in [M]. As a result, with new methods we have also improved the results that so far have been an ingredient in studying the Korenblum conjecture for the standard Bergman space, see [Ko], [KORZ], [KR], and [S]. The techniques to date have made use of the totally monotone functions. The direct connection of the present construction to the conjecture was discussed in [A]. As an added benefit the method happens to produce a new example related to the complex method of interpolation. This is explained in the last section.

\section{Domination and restriction}

DEFINITION 1.1. Say that a measure $\kappa$ on $\Omega$ is a contractive Carleson measure lir $L_{u}^{2}$ if for every $f \in L_{a}^{2}$ its norm is controlled by $\|f\|_{L^{2}(\Omega . \kappa)} \leq\|f\|$, that is,

$\left.i \quad(z)\right|^{2} d \kappa(z) \leq \int|f(z)|^{2} d \mu(z) ; \kappa$ is said to be saturated in the case that equality can ixcur.

With Theorem $A$ in view, the case of radially finitely supported measures is useful.

DEFivition 1.2. We say that $\lambda=\sum_{j=1}^{l} A_{j} \delta_{r_{j}}$, where $A_{j} \geq 0$ are real and $\delta_{r_{j}}$ is the point mass at $r_{j}>0$, is a contractive restriction measure if $\left\|M_{f}\right\|_{L^{2}(\lambda)}$ is at most $f$. that is, if for all $f \in L_{a}^{2}$

$$
\sum_{j=1}^{l} A_{j} \int_{|z|=r_{j}}|f(z)|^{2} \frac{d \theta}{2 \pi} \leq \int_{\Omega}|f(z)|^{2} d \mu(z) .
$$

PROPOSITION 1.3. A rotationally symmetric measure $\kappa$ is a contractive Carleson measure if and only if $\int_{\Omega}\left|z^{n}\right|^{2} d \kappa(z) \leq \int_{J_{\Omega}} x^{n} d v_{2}(x)$ for all $n \geq 0$. It is saturated precisely in the case that equality occurs for some $n$.

In particular $\lambda=\sum_{j=1}^{l} A_{j} \delta_{r_{j}}$ is a contractive restriction measure if and only iffor all $n \in \mathbb{Z}$

$$
\sum_{j=1}^{l} A_{j} r_{j}^{2 n} \leq \int_{J_{\Omega}} x^{n} d \nu_{2}(x) .
$$


The proof is easily obtained.

It is more convenient to construct the restriction measure using notation inherent in $v_{2}$. We will hence be mainly concerned with the relation

$$
\sum_{j=1}^{l} A_{j} x_{j}^{n} \leq \int_{J_{\Omega}} x^{n} d \nu_{2}(x) .
$$

OBSERVATION 1.4. Suppose that given the measure $\nu_{2}$ and an integer $l \geq 1$, it is possible to determine $A_{j}>0, j=1, \ldots, l$, and $x_{j}$ in the interior of $J_{\Omega}$ such that (1.1) holds for all $n \in \mathbb{Z}$. Then $r_{j}=\sqrt{x_{j}}$ and $A_{j}$ produce a contractive restriction measure for $d \mu=d \theta \otimes d \nu_{2}$ as defined in the introduction.

This is a straightforward consequence of the material so far.

The strategy to follow from now on is this. We fix a positive measure $\nu_{2}$ on $[0, \infty[$ and strive to make (1.1) valid for every $n$. However, we will add more demands on $\left\{x_{j}\right\},\left\{A_{j}\right\}$. They must also be such that (1.1) turns into equality for all $n$ in $\{k, k+1 \ldots, k+2 l-1\}$, where we specify $k$ in advance. Provided the construction is successful, Theorem A carries over to the present setting.

In fact, this will be achieved using the polynomials $\left\{p_{l}\right\}_{l=0}^{\infty}$ orthogonal with respect to $d \alpha_{k}(x)=x^{k} d \nu_{2}(x)$. The nodes $\left\{x_{j}\right\}_{1}^{l}$ will be the zeros of $p_{l}(x)$ and the weights $\left\{A_{j}\right\}_{1}^{l}$ will be the slightly modified Christoffel numbers of order $l$ for $\alpha_{k}$.

DEFINITION 1.5. Let $m_{n}$ denote the nth moment: $m_{n}=\int x^{n} d \nu_{2}(x)$. It is easily realized that $m_{n}=\left\|z^{n}\right\|^{2}=\int_{\Omega}\left|z^{n}\right|^{2} d \mu(z)$.

The single radius case is simple enough to be demonstrated at once.

PROPOSITION 1.6. Fix $k \in \Gamma_{\mu}$, with $k+1 \in \Gamma_{\mu}$, and put $l=1, x_{1}=m_{k+1} / m_{k}$, as well as $A_{1}=m_{k}^{k+1} / m_{k+1}^{k}$. Then (1.1) holds for all $n \in \mathbb{Z}$. This means that for all $f \in L_{a}^{2}$ we have

$$
\frac{m_{k}^{k+1}}{m_{k+1}^{k}} \int_{|z|=\sqrt{m_{k+1} / m_{k}}}|f(z)|^{2} \frac{d \theta}{2 \pi} \leq\|f\|_{L_{a}^{2}(\Omega, \mu)}^{2} .
$$

In addition, equality holds only for $f(z)=a_{k} z^{k}+a_{k+1} z^{k+1}$, all $a_{k}, a_{k+1} \in \mathbb{C}$.

PROOF. We need to prove that (1.1) holds and that equality occurs only for $n=k$ and $n=k+1$. It suffices to assume $n \in \Gamma_{\mu}$.

Starting with equality for $n=k, k+1,(1.1)$ demands

$$
\left\{\begin{array}{c}
A_{1} x_{1}^{k}=m_{k} \\
A_{1} x_{1}^{k+1}=m_{k+1}
\end{array}\right\} \Leftrightarrow\left\{\begin{array}{c}
x_{1}=m_{k+1} / m_{k} \\
A_{1}=m_{k}^{k+1} / m_{k+1}^{k}
\end{array}\right\} \text {. }
$$


With this choice, (1.1) for arbitrary $n$ requires

$$
A_{1} x_{1}^{n} \leq m_{n} \Leftrightarrow m_{k+1}^{n-k} \leq m_{n} m_{k}^{n-k-1} .
$$

For each $n>k+1$ Hölder's inequality with $p=n-k, p^{\prime}=(n-k) /(n-k-1)$ yields

$$
m_{k+1} \leq\left(\int x^{n p /(n-k)} d \nu_{2}(x)\right)^{1 / p}\left(\int x^{k(n-k-1) p^{\prime} /(n-k)} d \nu_{2}(x)\right)^{1 / p^{\prime}}=m_{n}^{1 /(n-k)} m_{k}^{(n-k-1) /(n-k)}
$$

which is (1.2). For $n<k$ the condition (1.2) takes the form $m_{k}^{k+1-n} \leq m_{n} m_{k+1}^{k-n}$ and Hölder's inequality is applied analogously with $p=k+1-n$ and $p^{\prime}=(k+1-$ $n) /(k-n)$. The claim for equality follows from the well known conditions on equality in Hölder's inequality. This finishes the proof.

REMARK. This proposition is the one closest to [M]. The entities $x_{1}$ and $A_{1}$ will reappear below for the polynomial of first degree connected to $d \alpha_{k}=x^{k} d \nu_{2}$.

\section{Quadrature formulas with poles}

This section begins with the facts on orthogonal polynomials to be used in the construction.

Again we fix $k \in \Gamma_{\mu}$ and consider $d \alpha_{k}(x)=x^{k} d v_{2}(x)$ on [0, $\infty$ [. Let $\left\{p_{n}\right\}_{n=0}^{\infty}$ be a sequence of polynomials obtained by successive orthogonalization of $1, x, x^{2}, \ldots$ with respect to $\alpha_{k}$. The polynomial $p_{n}$ of degree $n$ is unique up to a constant factor, which we will choose presently.

PROPOSITION 2.1. The polynomials $\left\{p_{n}\right\}_{n=0}^{\infty}$ given by

$$
p_{n}(x)=\left|\begin{array}{cccc}
1 & x & \ldots & x^{n} \\
m_{k} & m_{k+1} & \ldots & m_{k+n} \\
\vdots & \vdots & \ddots & \vdots \\
m_{k+n-1} & m_{k+n} & \ldots & m_{k+2 n-1}
\end{array}\right|
$$

are orthogonal with respect to $\alpha_{k}$.

PROOF. It suffices to prove $\int x^{l} p_{n}(x) d \alpha_{k}(x)=0$ for $l=0, \ldots, n-1$. The integral is readily rewritten as

$$
\left|\begin{array}{cccc}
m_{k+l} & m_{k+l+1} & \ldots & m_{k+l+n} \\
m_{k} & m_{k+1} & \ldots & m_{k+n} \\
\vdots & \vdots & \ddots & \vdots \\
m_{k+n-1} & m_{k+n} & \ldots & m_{k+2 n-1}
\end{array}\right|
$$

which obviously is zero for all $l=0, \ldots, n-1$. 
From now on we fix the orthogonal system $\left\{p_{n}\right\}_{n=0}^{\infty}$ according to Proposition 2.1. We will for each $k$ deal with only this choice.

PROPOSITION 2.2 ([F, Satz I.2.2]). Every $p_{n}$ has exactly $n$ real and simple zeros. If $v_{2}$ has support in $[0, R[, R=\infty$ allowed, then all zeros lie in $] 0, R[$.

The proof hinges only on the orthogonality and not on the representation as a determinant. The standard demonstration is omitted here.

Our way to establish (1.1) is now set.

PROPOSITION 2.3. Let $\nu_{2}$ be a measure on $\left[0, \infty\left[\right.\right.$ and put $\Gamma=\left\{n \in \mathbb{Z} ; \int x^{n} d \nu_{2}(x)\right.$ $<\infty\}$. Furthermore, take integers $k \in \Gamma$ and $l \geq 1$ such that $k+2 l-1 \in \Gamma$. Then there are explicit points $\left\{x_{j}\right\}_{j=1}^{l}$ in $] 0, \infty\left[\right.$, and positive numbers $\left\{A_{j}\right\}_{j=1}^{l}$ such that the following formula holds. For every $n \in \Gamma$

$$
\int x^{n} d v_{2}(x) \geq \sum_{j=1}^{l} A_{j} x_{j}^{n} .
$$

Equality holds precisely in the case that $n$ belongs to $\{k, k+1, \ldots, k+2 l-1\}$.

The proof will be conducted in three steps.

(i) Existence of real $A_{j}$.

Recall the technique of Hermitian polynomial interpolation:

For every set $\left\{y_{j}\right\}_{1}^{m}$ of nodes with multiplicities $\left\{d_{j}\right\}_{1}^{m}$, where $\sum_{i}^{m} d_{j}=n$, and for every smooth function $f$ there exists a polynomial $P_{n}(x ; f)$ of degree at most $n-1$ such that

$$
P_{n}^{(i)}\left(y_{j} ; f\right)=f^{(i)}\left(y_{j}\right), \quad 1 \leq j \leq m, 0 \leq i<d_{j} .
$$

For polynomials $f$ of degree at most $n-1$ the relation $P_{n}(\cdot ; f)=f$ holds.

This is proved in [Kr, pages 45-49] and [F, Hilfssatz I.1.3].

We first take the measure $d \alpha_{k}(x)=x^{k} d \nu_{2}(x)$, whose set of finite moments is $-k+\Gamma$. In particular, the moments of order $0, \ldots, 2 l-1$ are finite. Let $p_{l}$ be the $l$ th degree polynomial orthogonal with respect to $\alpha_{k}$ and take $\left\{x_{j}\right\}_{j=1}^{\prime}$ to be its zeros according to Proposition 2.2.

We now consider the interpolation nodes $\left\{x_{j}\right\}_{j=1}^{l}$ and multiplicities $d_{j}=1$. The corresponding Lagrange interpolation polynomial is of degree $l-1$ :

$$
P_{l-1}(x ; f)=\sum_{j=1}^{l} \beta_{j}(x) f\left(x_{j}\right) .
$$


Integrating this we find a quadrature formula exact for polynomials of degree at most $l-1$ :

$$
\int f(x) x^{k} d \nu_{2}(x)=\sum_{j=1}^{l} A_{j} x_{j}^{k} f\left(x_{j}\right)
$$

The constants are for notational convenience chosen with factors $x_{j}^{k}$. Next, (2.3) must hold for degrees not greater than $2 l-1$. Every such polynomial $r$ is given by $r(x)=r_{1}(x)+p_{l}(x) r_{2}(x)$, where $r_{1}$ and $r_{2}$ have degrees not exceeding $l-1$. From Proposition 2.1 it follows that $\int p_{l}(x) r_{2}(x) x^{k} d \nu_{2}(x)=0$, whence $\int r(x) x^{k} d \nu_{2}(x)=$ $\int r_{1}(x) x^{k} d \nu_{2}(x)$. On the other hand, the value of the right-hand side in (2.3) is the same for $f=r$ and $f=r_{1}$.

(ii) Positivity of $A_{j}$.

Put $r_{j}(x)=\left[p(x) /\left(x-x_{j}\right)\right]^{2}$, which is of degree $2 l-2$. Using (2.3)

$$
0<\int r_{j}(x) x^{k} d \nu_{2}(x)=A_{j} x_{j}^{k} p^{\prime}\left(x_{j}\right)^{2},
$$

and consequently $A_{j}$ is strictly positive.

(iii) The inequality for $n<k$ and $n \geq k+2 l$.

Take $n \in \Gamma$ with $n<k$. Let $Q_{n}(x)$ be the polynomial specified by Hermitian interpolation:

$$
\begin{cases}Q_{n}\left(x_{j}\right)=x_{j}^{n-k}, & j=1, \ldots, l, \\ Q_{n}^{\prime}\left(x_{j}\right)=(n-k) x_{j}^{n-k-1}, & j=1, \ldots, l .\end{cases}
$$

Consider $R_{n}(x)=x^{n-k}-Q_{n}(x)$, which has zeros of multiplicity two at each $x_{j}$, and is smooth in ]0, $\infty$ [. In addition, $R_{n}^{(2)}(x)>0$ on the right half-line, whence $R_{n}^{(2)-1)}$ has at most one zero there. Then $R_{n}^{(21-2)}$ can have at most two zeros in ]0, $\infty[$. Repetition of this argument proves that $R_{n}$ itself has at most $2 l$ zeros counting multiplicity. The construction of $Q_{n}$ now forces $2 l$ to be the exact number. The zero at every $x_{j}$ has exact order two, and since $\lim _{x \rightarrow 0^{+}} R_{n}(x)=+\infty$, we find $R_{n} \geq 0$ in ]0, $\infty$, that is, $x^{n-k} \geq Q_{n}(x)$ on the right half-line. Next, $Q_{n}(x)$ has degree $2 l-1$, whence application of (2.3) and (2.4) shows that

$$
\int x^{n} d \nu_{2}(x)>\int x^{k} Q_{n}(x) d \nu_{2}(x)=\sum_{j=1}^{l} A_{j} x_{j}^{k} Q_{n}\left(x_{j}\right)=\sum_{j=1}^{l} A_{j} x_{j}^{n} .
$$

The strict inequality is due to the assumption that the support is not finite.

For the remaining case $n \geq k+2 l$ the argument is the same, except that now $\lim _{x \rightarrow+\infty} R_{n}(x)=+\infty$ is used in order to gain $R_{n}(x) \geq 0$ on the right half-line. This completes the proof of Proposition 2.3. 
REMARK. The proof of the above inequalities has been influenced by [F, Hilfssatz III.1.5]. Such results were first found by Shohat.

\section{Restriction operators}

The careful formulation of the result in the preceding section is as follows.

THEOREM 3.1. Let $\mu$ be a rotationally symmetric measure on $\mathbb{C}$ with radial component measure $\nu_{2}$. Fix integers $l \geq 1$ and $k \in \Gamma_{\mu}$ such that $k+2 l-1 \in \Gamma_{\mu}$. Denote by $x_{1}, \ldots, x_{l}$ the zeros of the lth polynomial orthogonal with respect to $x^{k} d v_{2}(x)$. Determine the (positive) numbers $A_{1}, \ldots, A_{l}$ through the equations $\sum_{j=1}^{\prime} A_{j} x_{j}^{n}=\int x^{n} d \nu_{2}(x), n=k, \ldots, k+l-1$. Then the norm on $L_{a}^{2}(\mu)$ has the property that, for $r_{j}=\sqrt{x_{j}}$ and all $f \in L_{a}^{2}(\mu)$,

$$
\sum_{j=1}^{l} A_{j} \int_{|z|=r_{j}}|f(z)|^{2} \frac{d \theta}{2 \pi} \leq\|f\|^{2} .
$$

Moreover, equality holds precisely in case that $f$ is of the form $f(z)=a_{k} z^{k}+\cdots+$ $a_{k+2 l-1} z^{k+2 l-1}$.

DEFINITION 3.2. The Jacobi restriction measure for $\mu$ with parameters $k \in \Gamma_{\mu}$, $l \geq 1$, is the measure $\lambda=\lambda_{k l}(\mu)$ on $\mathbb{C}$ specified by: $\lambda$ has support $\bigcup_{j=1}^{l}\left\{|z|=r_{j}\right\}$ and is uniform of mass $A_{j}$ on each circle $\left\{|z|=r_{j}\right\}$, where $r_{j}$ and $A_{j}$ are taken from Theorem 3.1.

This concept of Jacobi restriction measure conforms to Definition 1.2, as we see from Theorem 3.1. Using a natural extension of Definition 1.1 we formulate the following theorem.

THEOREM 3.3. The Jacobi restriction measure $\lambda=\lambda_{k l}$ for $\mu$ is a contractive Carleson measure for $L_{a}^{2 m}(\Omega, \mu)$, where $m$ is a positive integer, that is, for all $f$ in $L_{a}^{2 m}(\Omega, \mu)$ the inequality $\|f\|_{L^{2 m}(\lambda)} \leq\|f\|_{L_{a}^{2 m}(\Omega, \mu)}$ obtains. In addition, norm equality holds exactly when $f(z)^{m}=a_{k} z^{k}+\cdots+a_{k+2 l-1} z^{k+2 l-1}$.

The proof is simply an application of Theorem 3.1 to the analytic function $f(z)^{m}$ in $L_{a}^{2}$, which appears for every $f \in L_{a}^{2 m}(\Omega, \mu)$.

By variation of the parameters $k$ and $l$ we can even get a partial ordering of the corresponding Jacobi restriction measures.

THEOREM 3.4. Suppose that $k \leq p<p+2 q-1 \leq k+2 l-1$. Then

$$
\|f\|_{L^{2 m}\left(\lambda_{p q}\right)} \leq\|f\|_{L^{2 m}\left(\lambda_{k l}\right)} \leq\|f\|_{L_{a}^{2 m}(\mu)}
$$

holds for all $f$ analytic on $\Omega$. Furthermore, the first equality holds precisely when the two outer members are equal. 
The technique used so far to compute moments reduces the proof to the demonstration of:

PROPOSITION 3.5. Assume the relation $k \leq p<p+2 q-1 \leq k+2 l-1$. Denote the data for the restriction measure $\lambda_{k l}=\lambda_{k l}(\mu)$ by $\left\{A_{j}\right\}_{j=1}^{l},\left\{r_{j}=\sqrt{x_{j}}\right\}_{j=1}^{l}$, and those for $\lambda_{p q}=\lambda_{p q}(\mu)$ by $\left\{B_{j}\right\}_{j=1}^{q},\left\{s_{j}=\sqrt{y_{j}}\right\}_{j=1}^{q}$. Then for every $n \in \mathbb{Z}$, the nth moments satisfy

$$
\sum_{j=1}^{q} B_{j} y_{j}^{n} \leq \sum_{j=1}^{l} A_{j} x_{j}^{n} .
$$

Equality holds only for $n=p, p+1, \ldots, p+2 q-1$.

PROOF. It suffices to prove the case $q=l-1$ and the subcases $p=k$ and $p=k+1$. The general case is proved by several applications of these reduced cases; in fact, as long as $k<p$ or $q<l-1$. Both of the subcases will be handled simultaneously. Hence we assume $q=l-1$ as well as $p=k$ or $p=k-1$. Write

$$
K_{1}(t)=\sum_{j=1}^{t} A_{j} x_{j}^{t}, \quad K_{2}(t)=\sum_{j=1}^{t-1} B_{j} y_{j}^{t} .
$$

The original construction yields $K_{1}(n)=\int x^{n} d \nu_{2}(x)$, for $n=k, \ldots, k+2 l-1$ as well as $K_{2}(n)=\int x^{n} d v_{2}(x)$, for $n=p, \ldots, p+2 l-3$. In particular, $K_{1}(t)-K_{2}(t)$ has zeros at $t=p, \ldots, p+2 l-3$, that is, at least $2 l-2$ zeros. We need the following lemma which is a special case of [PS, section V, problem 75].

LEMMA 3.6. Any real equation $b_{1} e^{a_{1} x}+\cdots+b_{m} e^{a_{m} x}=0$ has at most $m-1$ real zeros.

From the lemma we conclude that $K_{1}(t)-K_{2}(t)$ has exactly $2 l-2$ zeros, namely the points $t=p, \ldots, p+2 l-3$. From Section 2 we have $K_{2}(p-1)<\int x^{p-1} d v_{2}(x)$ and $K_{2}(p+2 l-2)<\int x^{p+2 l-2} d v_{2}(x)$. In case $p=k+1$ this means that $K_{1}(k)-K_{2}(k)>$ 0 and in case $p=k$ we have $K_{1}(k+2 l-2)-K_{2}(k+2 l-2)>0$. Since the zeros are distinct and even in number, $K_{1}(t)-K_{2}(t)>0$ outside $[p, p+2 l-3]$ in both subcases. Hence $K_{1}(n) \geq K_{2}(n)$ for all $n \in \mathbb{Z}$ and equality holds only for $n=p, \ldots, p+2 l-3$. The proof is complete.

REMARK. The partial ordering above is, in fact, cofinal: given two restriction measures we just choose parameters of a third measure so as to make it exact for at least all the moments for which either of the two given measures are exact. Obviously this net of measures converges to the original measure $\mu$ throughout $\Omega$. 


\section{Harmonic functions}

Not all of the preceding results are true if one relaxes the assumption on analyticity to only harmonicity. This will be demonstrated by counterexamples as well as by new proofs where something can be rescued. We will write $L_{h}^{2}(\Omega, \mu)$ for the space of complex valued harmonic functions in $L^{2}(\Omega, \mu)$.

PROPOSITION 4.1. Suppose $\Omega$ is a disk and that $g$ is harmonic in $\Omega$. Then for all parameters $k$ and $l$ the inequality $\|g\|_{L^{2}(\lambda, k)} \leq\|g\|_{L_{i,}^{2}(\mu)}$ holds. Hence the Jacobi restriction measure is a contractive Carleson measure for $L_{h}^{2}(\Omega, \mu)$.

PROOF. Any function harmonic in a zero-centered disk can be represented $g\left(r e^{i \theta}\right)$ $=\sum_{n=-\infty}^{\infty} a_{n} r^{|n|} e^{i n \theta}$. Then we find $\int_{|z|=\sqrt{x}}|g(z)|^{2} d \theta / 2 \pi=\sum_{n=-\infty}^{\infty}\left|a_{n}\right|^{2} x^{|n|}$ due to orthogonality. This means we may apply the reasoning from the analytic case to deduce

$$
\|g\|_{L_{h}^{2}(\Omega, \mu)}^{2}=\int\left(\sum_{n=-\infty}^{\infty}\left|a_{n}\right|^{2} x^{|n|}\right) d v_{2}(x) \geq \sum_{j=1}^{l} A_{j} \int_{|z|=\sqrt{x_{j}}}|g(z)|^{2} \frac{d \theta}{2 \pi}=\|g\|_{L^{2}\left(\lambda_{k H}\right)}^{2} .
$$

PROPOSITION 4.2. Let $g$ be harmonic in $\Omega$. Assume that $\int_{|z|=r} g(z) d \theta / 2 \pi$ is independent of $r$ and that $0 \in \Gamma_{\mu}$, that is, $\mu(\Omega)<\infty$. For all parameters $k$ and $l$ such that $k \leq 0 \leq 2 l+k-1$, the norm inequality $\|g\|_{L^{2}\left(\lambda_{k l}\right)} \leq\|g\|_{L_{h}^{2}(\mu)}$ holds.

PROOF. The assumption on $g \in L^{2}(\mu)$ implies a representation

$$
g\left(r e^{i \theta}\right)=\sum_{n=-\infty}^{\infty}\left(a_{n} r^{n}+b_{n} r^{-n}\right) e^{i n \theta}
$$

It follows that

$$
\int_{|z|=\sqrt{x}}|g(z)|^{2} \frac{d \theta}{2 \pi}=\sum_{n=-\infty}^{\infty}\left(\left|a_{n}\right|^{2} x^{n}+\left|b_{n}\right|^{2} x^{-n}+2 \operatorname{Re} a_{n} \bar{b}_{n}\right) .
$$

Since $k \leq 0 \leq 2 l+k-1$, we know that the general relation $\int x^{n} d v_{2}(x) \geq \sum_{j=1}^{l} A_{j} x_{j}^{n}$ is an equality for $n=0$. On the grounds that every coefficient in (4.1) with $n \neq 0$ is non-negative, a termwise integration yields

$$
\int_{\Omega}|g(z)|^{2} d \mu(z) \geq \int|g(z)|^{2} d \lambda_{k l}(z),
$$

which is the claimed property. 
EXAMPLE 4.3. We construct two instances where relaxation of the conditions in Proposition 4.2 produces the reversed inequality.

Consider any ring domain and any accompanying $\nu_{2}$ with $\int x^{n} v_{2}(x)<\infty$ for $n=-2,-1,0,1,2$. Choose $a>0$ such that $\left.J_{\Omega} \subseteq\right] 3 / 2 a, \infty[$. It is claimed that for the parameters $l=1$ and $k=1$ there is a harmonic function $g \in L_{h}^{2}(\Omega, \mu)$ such that $\|g\|_{L_{h}^{2}(\mu)}<\|g\|_{L^{2}\left(\lambda_{11}\right)}$. In fact, $g(z)=a z-1 / \bar{z}$ is a good choice.

Put $F(x)=x^{-1} \int_{|z|=\sqrt{x}}|g(z)|^{2} d \theta / 2 \pi=x^{-1}\left(a^{2} x-2 a+1 / x\right)$. The choice of $a$ yields $F^{\prime}(x)>0$ and $F^{\prime \prime}(x)<0$ throughout $] 3 / 2 a, \infty[$. We now use the technique from Section 2. Consider the one point quadrature formula for $x d \nu_{2}(x)$ specified by $A_{1}$ and $x_{1}$. Let $Q(x)=F^{\prime}\left(x_{1}\right)\left(x-x_{1}\right)+F\left(x_{1}\right)$ be the Hermite interpolant at $x=x_{1}$ of order two. Then $F(x)-Q(x)$ has only one double zero in $] 3 / 2 a, \infty[$ and no other zeros. In fact, $F(x) \leq Q(x)$ throughout $] 3 / 2 a, \infty\left[\right.$ with strict inequality for $x \neq x_{1}$. Integrating, we find (with the usual non-finiteness assumption on the support)

$$
\int F(x) x d \nu_{2}(x)<\int Q(x) x d v_{2}(x)=A_{1} x_{1} Q\left(x_{1}\right)=A_{1} x_{1} F\left(x_{1}\right) .
$$

The definition of $F(x)$ finally yields

$$
\int_{\Omega}|g(z)|^{2} d \mu(z)<A_{1} x_{1} F\left(x_{1}\right)=A_{1} \int_{|z|=\sqrt{x_{1}}}|g(z)|^{2} \frac{d \theta}{2 \pi} .
$$

Using a more intricate calculation we can get also $\|g\|_{L^{2}(\mu)}<\|g\|_{L^{2}\left(\lambda_{12}\right)}$, which is an example with two circles. Let $A_{1}, A_{2}, x_{1}, x_{2}$, be the data for $\lambda_{12}$, and take $Q_{2}(x)$ to be the Hermite interpolant for the previous $F(x)$ with nodes $x_{1}, x_{2}$, and orders $d_{j}=2$. One finds that $Q_{2}(x)=2\left(x_{2}-x_{1}\right)^{-2}\left(x_{1}^{-1}-x_{2}^{-1}\right)^{2}\left(a-x_{1}^{-1}-x_{2}^{-1}\right) x^{3}+$ lower terms. Choosing $a$ so large that $\left.J_{\Omega} \subseteq\right] 5 / 2 a, \infty$, one finds that $F^{(4)}(x)<0$ and $F^{\prime \prime \prime}(x)>0$ on ]5/2a, $\infty$ [. As before, the inequality $F(x) \leq Q_{2}(x)$ holds on $J_{\Omega}$ with equality only for $x=x_{1}, x_{2}$. Integrating this, $\int|g|^{2} d \mu<\int|g|^{2} d \lambda_{12}$ follows.

EXAMPLE 4.4. In the statement of Proposition 4.2 one contribution was excluded, namely the harmonic function $\log |z|$. Its inclusion will produce a further counterexample.

Consider $g(z)=\log a|z|$. Then $F(x)=\int_{|z|=\sqrt{x}}|g|^{2} d \theta / 2 \pi$ can, by a proper choice of $a$, give $F^{\prime \prime}(x)<0, F^{\prime}(x)>0$ on any fixed $[\epsilon, \infty$. Calculations similar to Example 4.3 will prove that $\|g\|_{L_{h}^{2}(\mu)}<\|g\|_{L^{2}\left(\lambda_{01}\right)}$.

\section{The intermediary $L_{a}^{p}$-spaces}

It is natural to ask about the validity of the previous results for the spaces $L_{a}^{p}(\Omega, \mu)$. Some negative examples and positive results have been collected in this section. 
Consider the subspace $L_{t}^{p}(\Omega, \mu)$ being the closure of the analytic polynomials in $L_{a}^{p}(\Omega, \mu)$. Each such function has a Laurent expansion in $\Omega$ containing only powers with non-negative exponents. In particular, they can be analytically continued into the hole around the origin. which $\Omega$ encloses. Using the method of $[\mathrm{A}]$ we have a differentiability result:

PROPOSITION 5.1. For each $L_{f}^{p}(\Omega, \mu), p \geq 2 n-2$, the pth integral mean $M_{p} f(r)=\int_{|:|=, r}|f|^{r} d w^{\prime} 2$, has $n$ positive and continuous derivatives for $r$ less than the outer radius of $\Omega$

LEMMA 5.2. Let $1 \geq 1$ and $k \geq 0$. For any polynomial $Q$ with non-negative coefficients of each order not contained in $\{k, k+1, \ldots, 2 l+k-1\}$, a quadrature estimate holds: I $Q d_{1}:-\sum A, Q\left(x_{j}\right)$, where $x_{j}$ are the points constructed for $\lambda_{k l}$.

PROOF. Winte $Q(1)=\varphi(x)+x^{k} q_{2}(x)+x^{2 l+k} q_{3}(x)$, with $\operatorname{deg} q_{1} \leq k-1$ and $\operatorname{deg} q_{2} \leq 2 l-1$. He hnom that $\sum A_{j} q_{2}\left(x_{j}\right) x_{j}^{k}$ is equal to $\int q_{2}(x) x^{k} d \nu_{2}$ by construction. Furthermore. $\Sigma+1^{-}=i x^{n} d \nu_{2}$ for all integers $n$, so using the non-negativity of the coefficient $\ln _{4} \mathrm{and}_{4}$. the addition of all contributions including $q_{2}$ establishes the claim.

A result for general Bergmun spaces is given by the following proposition.

PROPOSTIOS S: Sur;w se the ring domain $\Omega$ is bounded and $l \geq 1, k \in \Gamma_{\mu}$. Put $\kappa=\max (0 . k)$ and wirm, thut $r \geq 4 l+2 \kappa-2$. Then every $f \in L_{t}^{p}(\Omega, \mu)$ satisfies for $\lambda=\lambda_{k l}$

$$
f\left\|_{L \sim(\dot{L})} \leq\right\| f \|_{L_{u}^{p}(\mu)} \text {. }
$$

REMARK. Our methind of proof is not suitable for functions with a proper Laurent expansion so they will te ignored for this result.

PROOF. Let $M(r)=M_{r} f(r)$ be the $p$ th integral mean of $f$. From Proposition 5.1 we know that $M \in C^{\prime \cdot+2}([0, R])$ and all derivatives are non-negative. Consider the Hermite interpolant $P(r)$ for $M(r)$ with nodes $x_{1}, \ldots, x_{l}$ of degree 2 and $x_{0}=0$ of degree $\kappa$. Following $[\mathrm{A}]$ or [ $\mathrm{Kr}$, pages 45-49] we have

$$
M(r)=P(r)+\frac{\omega(r)^{2} r^{\kappa}}{(\kappa+2 l) !} M^{(\kappa+2 l)}(\xi),
$$

where $\xi$ is in $[0, R]$ and $\omega(r)$ is the polynomial with zeros $x_{1}, \ldots, x_{n}$ and suitably normalized. Since no derivative of $M$ is negative, we know that the terms of orders $0,1, \ldots, \kappa-1$ in $P(r)$ are non-negative. In the case $k \geq 1$ we may invoke Lemma 5.2. 
The non-negative contribution from the remainder term is unproblematic and we conclude that

$$
\|f\|_{L^{p}(\lambda)}^{p}=\int M(|\cdot|) d \lambda \leq \int M d \nu_{2}=\|f\|_{L_{L}^{p}(\mu)}^{p} .
$$

For the possibility $\kappa=0$, that is, $k \leq 0$, a result similar to Lemma 5.2 but with exactness for the terms of order $\{0, \ldots, 2 l+k-1\}$ (or for none at all when $k<-2 l+1$ ) is incorporated to yield the same conclusion.

Presently we will produce examples showing that the range of the exponent $p$ is optimal to some extent, but first we will reinterpret the method in the way it depends on convexity. This will produce an ample supply of counterexamples.

After a change of measure the partial ordering at the end of Section 3 says that if $0 \leq n<2 l-1$, then the inequality $\int|f|^{2} d \lambda_{n 1} \leq \int|f|^{2} d \lambda_{0}$ holds. To see the meaning of this relation we let $g(x)$ be an arbitrary function for which the inequality $\int g d \lambda_{n 1} \leq \int g d \lambda_{01}$ holds. Denote the data for $\lambda_{01}$ by $x_{j}, A_{j}$ and those for $\lambda_{n 1}$ by $y_{1}$, $B_{1}$. Our inequality claims that

$$
B_{1} g\left(y_{1}\right) \leq \sum_{j=1}^{l} A_{j} g\left(x_{j}\right) .
$$

However, we also know that

$$
\begin{aligned}
B_{1} y_{1}^{n} & =\int x^{n} d \nu_{2}=\sum A_{j} x_{j}^{n}, \\
B_{1} y_{1}^{n+1} & =\int x^{n+1} d \nu_{2}=\sum A_{j} x_{j}^{n+1} .
\end{aligned}
$$

Observe first the representation

$$
y_{1}=\sum_{j} \frac{A_{j} x_{j}^{n}}{\sum_{k} A_{k} x_{k}^{n}} x_{j}
$$

which expresses $y_{1}$ as a convex combination of all $x_{j}$. Next, (5.1) becomes

$$
\frac{g\left(y_{1}\right)}{y_{1}^{n}} \leq \sum_{j} \frac{A_{j} x_{j}^{n}}{B_{1} y_{1}^{n}} \frac{g\left(x_{j}\right)}{x_{j}^{n}}=\sum_{j} \frac{A_{j} x_{j}^{n}}{\sum_{k} A_{k} x_{k}^{n}} \frac{g\left(x_{j}\right)}{x_{j}^{n}},
$$

where the right-hand side has the same convex combination as $y_{1}$ has.

OBSERVATION 5.4. (1) For functions $g$ such that $x^{-n} g(x)$ is convex, the inequality $\int g d \lambda_{n 1} \leq \int g d \lambda_{0 l}$ always holds.

(2) In the case that $x^{-n} g(x)$ is non-convex, the inequality in (1) is false as soon as the interval $\left[x_{1}, x_{l}\right]$ is an interval of concavity for this function. 
Interpreting this for our integral means, we have established a geometrically improved result, related to Theorem 3.4:

ProposItION 5.5. Suppose that $k \leq n<k+2 l-1, p \geq 2$. Then

$$
\|f\|_{L^{p}\left(\lambda_{n 1}\right)} \leq\|f\|_{L^{p}\left(\lambda_{k 1}\right)}, \quad\|f\|_{L^{p}\left(\lambda_{n}\right)} \leq\|f\|_{L_{a}^{p}(\mu)}
$$

hold for all measures $\mu$ and all $f$ analytic on $\Omega$ such that $x^{-n} M_{p} f(x)$ is convex. For each $f$ giving non-convexity of $x^{-n} M_{p} f(x)$ there are measures $\mu$ simultaneously negating both inequalities for this particular function $f$.

The proof is entirely contained in Observation 5.4 except the result for $\lambda_{n 1}$ and $\mu$. This involves one radius and a quadrature formula exact for linear polynomials. The relation of the tangent to (respectively) a convex and non-convex curve is all that is needed for the integration to produce the claimed result. Observe also that for $p$ an even integer convexity always results and this explains the previous success.

Now we can produce illuminating counterexamples.

EXAMPLE 5.6 (Sharpness of Proposition 5.3 with offset restriction). Take a nonnegative integer $n$ and consider $L_{a}^{p}(\Omega, \mu)$ with the only condition $2 n<p<2 n+2$. The Jacobi restriction measure $\lambda=\lambda_{n 1}(\mu)$ will produce the required example.

The polynomial $f(z)=z$ has normalized integral mean $x^{-n} M_{p} f(x)=x^{p / 2-n}$, which is strictly concave on the positive axis. By Proposition 5.5 and Observation 5.4 (2) the inequality $\|z\|_{L^{p}(\lambda)}>\|z\|_{L_{a}^{p}(\Omega, \mu)}$ holds for any measure $\mu$ on $\Omega$. At the same time we find that for any other restriction measure we have $\|z\|_{L^{p}\left(\lambda_{n_{1}}\right)}>\|z\|_{L^{p}\left(\lambda_{k}\right)}$ as soon as $k \leq n<k+2 l-1$.

REMARK. Probably it is not worth the effort to try to construct counterexamples negating only one of the inequalities in Proposition 5.5.

EXAMPLE 5.7 (Sharpness of Proposition 5.3 for $l$ radii). Let $l \geq 1$ and suppose that $4(l-1)<p<4 l-2$. Our claim is that $\|z\|_{L^{r}(\mu)}<\|z\|_{L^{p}(\lambda)}$ for the Jacobi restriction measure $\lambda=\lambda_{0 \text { l }}$ independently of $\mu$. In fact, for $f(z)=z$ we have $d^{2 l} M_{p} f(r) / d r^{2 l}$ negative on $] 0, \infty\left[\right.$, since $M_{p} f(r)=r^{p / 2}$ and $2 l-2<p / 2<$ $2 l-1$. Let $Q(r)$ be the Hermite interpolant with $l$ nodes of multiplicity two at each $x_{j}$ corresponding to a radius in the construction of $\lambda_{0 l}$. Since $Q$ has degree $2 l-1$, which is greater than the power $p / 2$ occurring in $M_{p} f(r)$, the observation $d^{2 l}\left\{M_{p} f(r)-Q(r)\right\} / d r^{2 l}<0$ everywhere suffices to prove $M_{p} f \leq Q$ with equality only at $l$ points. The radially infinite support of $\mu$ then forces the claimed norm inequality. 
Finally, let us cast one aspect of the material in a slightly different form. Theorem 3.3 says that the restriction operator $\mathscr{R}_{k l}$ has operator norm 1 from $L_{a}^{2 m}(\mu, \Omega)$ to $L^{2 m}\left(\lambda_{k l}, \cup_{1}^{l}\left\{|z|=r_{j}\right\}\right)$, for each integer $m$. Were each $L_{a}^{p}(\mu)$ the correct interpolating space with respect to the Riesz-Thorin interpolation, then the operator norm $\mathscr{P}_{k l}: L_{a}^{p}(\mu) \mapsto L^{p}\left(\lambda_{k l}\right)$ necessarily would have to be at most one for all $p \geq 2$. However, Examples 5.6 and 5.7 demonstrate that $\left\|\mathscr{R}_{k 1}\right\|_{p \rightarrow p}>1$ and $\left\|\mathscr{R}_{0 l}\right\|_{p \rightarrow p}>1$ for suitable $p$ in the relevant range. Thus the interpolation between a couple of Bergman spaces yields spaces not isometric to the correct space in the same scale of Bergman spaces. Likewise by the same examples, not even the complex method of interpolation with respect to the full family $\left\{L_{a}^{2 m}(\mu)\right\}_{m=1}^{\infty}$, in the sense of St. Louis spaces, isometrically preserves the Bergman spaces. This is so since the operator norm for St. Louis spaces obeys the same log-convexity property as expressed in the Riesz-Thorin theorem. Therefore we have found a new example consisting of analytic spaces illustrating the shortcomings of a complex method interpolation. For this particular operator $\mathscr{R}_{k l}$ the convexity of the integral means is governed by the expression $\sqrt{x}$ as wen above, whereas the log-convexity in general is incompatible with this notion of convexity. The logarithmic convexity is, however, the correct one in the three circles theorem of Hadamard and hence appears in the interpolation method.

\section{Addendum: higher dimensions}

The basic construction of the restriction measures $\lambda_{k l}$ may be used also for harmonic tunitions in higher dimensional euclidean spaces. Only one result is stated here in irder to present the mechanisms.

$W_{e}$ consider measures $\mu$ on $\mathbb{R}^{d}$ invariant with respect to the action of $\operatorname{SO}\left(\mathbb{R}^{d}\right)$. The ve measures can be decomposed into a radial component and a normalized surface measure on the unit sphere, in a manner imitating the decomposition in the complex plane. The definition of the Jacobi restriction measure $\lambda_{k l}(\mu)$ is reinterpreted as producing a measure supported on the union $\bigcup_{j=1}^{l}\left\{x \in \mathbb{R}^{d}:|x|=r_{j}\right\}$. On each component $\left\{x \in \mathbb{R}^{d}:|x|=r_{j}\right\}$ the restriction of $\lambda_{k l}$ is a multiple $A_{j}$ of the normalized surface measure. This measure $\lambda_{k l}$ exists precisely when $\int|x|^{2 k} d \mu(x)$ and $\int|x|^{2(k+2 l-1)} d \mu(x)$ are finite.

Proposition. Let $\Omega \subseteq \mathbb{R}^{d}$ be a ball centered at the origin. Suppose further that $k \leq p<p+2 q-1 \leq k+2 l-1$ holds and that $\lambda_{k l}$ exists. Then every function $f$ harmonic in $\Omega$ satisfies

$$
\|f\|_{L^{2}\left(\lambda_{p q}\right)} \leq\|f\|_{L^{2}\left(\lambda_{k t}\right)} \leq\|f\|_{L^{2}(\mu)} .
$$

PROOF. Since $\lambda_{k l}$ exists, so does $\lambda_{p q}$ according to the condition on its parameters. 
Using the Laplace's series for $f$, we have $f \sim \sum_{m=0}^{\infty} r^{m} Y_{m}$, where $Y_{m} \in \mathscr{H}_{m}$ is homogeneous of degree $m$. It follows that for normalized surface measure $\sigma$

$$
\int_{|x|=1}|f(r x)|^{2} d \sigma(x)=\sum_{m=0}^{\infty}\left\|Y_{m}\right\|_{L^{2}(\sigma)}^{2} r^{2 m}
$$

Using Proposition 2.3 as well as Proposition 3.5 we get the claimed ordering of the three norms.

\section{References}

[A] M. E. Andersson, 'Integral means on Bergman spaces', Complex Variables Theory Appl. 132 (1997), 147-160.

[F] G. Freud, Orthogonale Polynome, Mathematische Reihe, Band 33 (Birkhäuser, Basel, 1969).

[Ko] B. Korenblum, 'A maximum principle for the Bergman space', Publ. Mat. 35 (1991), 479-486.

[KORZ] B. Korenblum, R. O' Neil, K. Richards and K. Zhu 'Totally monotone functions with applications to the Bergman space', Trans. Amer. Math. Soc. 337 (1993), 795-806.

[KR] B. Korenblum and K. Richards, 'Majorization and domination in the Bergman space', Proc. Amer. Math. Soc. 117 (1993), 153-158.

[Kr] V. Krylov, Approximate calculation of integrals (transl. A. Stroud) (Macmillan, New York, 1962).

[M] J. Matero, 'On Korenblum's maximum principle for Bergman space', Arch. Math. 64 (1995), 337-340.

[PS] G. Polya and G. Szegö, Aufgaben und Lehrsätze aus der Analysis II (Springer, Berlin, 1971).

[S] W. Schwick, 'On Korenblum's maximum principle', Proc. Amer. Math. Soc. 125 (1997), 2581-2588.

Department of Mathematics

Kungliga Tekniska Högskolan

Lindstedtsvägen 25

S-100 44 Stockholm

Sweden

e-mail: matsa@math.kth.se

Current address: Department of Mathematics

University of Stockholm

S-106 91 Stockholm

Sweden

e-mail: mats.andersson@matematik.su.se 